\title{
Diagnostic and therapeutic push type enteroscopy in clinical use
}

\author{
G R Davies, M J Benson, D J Gertner, R M N Van Someren, D S Rampton, C P Swain
}

\begin{abstract}
This study describes small bowel push enteroscopy in routine clinical practice, using a purpose designed instrument (Olympus SIF-10). Fifty six patients had a total of 60 procedures over a two and a half year period. The median (range) depth of small intestine intubated was 45 (15-90) cm. Procedure time varied from 10-45 minutes. Most enteroscopies were performed during routine gastroscopy lists. The technique was comparatively easy for experienced endoscopists to learn. Forty two procedures were for diagnostic purposes. Eleven patients had gastrointestinal bleeding where the source was obscure, or where early investigations had suggested a small bowel source: a specific diagnosis was made in $45 \%$ of these cases. Of seven iron deficient anaemic patients using non-steroidal anti-inflammatory drugs (NSAIDs), only one had a lesion detected in the upper small bowel. Nine patients had abnormal small bowel barium studies. Small bowel abnormalities were seen in six cases and were definitively diagnostic in three of these; in three patients the barium study appearances were confirmed as artefact. Fifteen patients were investigated for abdominal symptoms suggesting small bowel obstruction or malabsorption: a diagnosis was made in five cases. Fifteen patients underwent enteroscopy for therapeutic purposes, including successful treatment of difficult enteral feeding problems by nasojejunal tubes or by cutaneous endoscopic jejunostomies, polypectomy for Peutz-Jeghers syndrome, and dilatation of strictures. Additionally, bleeding lesions detected in patients during investigation of anaemia were successfully treated at the time by YAG
\end{abstract}

TABLE I Comparison of push and sonde type enteroscopy

\begin{tabular}{|c|c|c|}
\hline & Push & Sonde \\
\hline Model & Olympus SIF 10/10L & Olympus SIF-SW \\
\hline Working length & $168 / 200 \mathrm{~cm}$ & $270 \mathrm{~cm}$ \\
\hline Diameter & $11.2 \mathrm{~mm}$ & $5 \mathrm{~mm}$ \\
\hline Limit of small bowel accessible & Mid-distal jejunum & Distal ileum $(70-80 \%)$ \\
\hline Mucosa seen & About $100 \%$ & About $50-70 \%$ \\
\hline Procedure time & $10-45 \mathrm{~min}$ & $\begin{array}{l}\text { 12-24 h (unassisted) } \\
4-8 \mathrm{~h}\left(\text { assisted }^{\star}\right)\end{array}$ \\
\hline Fluoroscopy/extra staff required & Only for some therapeutic uses & Always \\
\hline Biopsy/therapy channel & $2 \cdot 8 \mathrm{~mm}$ & None \\
\hline Tip deflection & $\begin{array}{l}180^{\circ} \text { up/down } \\
160^{\circ} \text { left/right }\end{array}$ & None \\
\hline Field of view & $120^{\circ}$ forward & $90^{\circ}$ forward \\
\hline $\begin{array}{l}\text { Therapeutic procedures possible } \\
\text { Routine upper/lower endoscopy }\end{array}$ & Yes & No \\
\hline $\begin{array}{l}\text { Routine upper/lower endoscopy } \\
\text { possible with instrument? }\end{array}$ & Yes & No \\
\hline Patient discomfort & As for routine gastroscopy & Often considerable \\
\hline Cost & About $£ 18000$ & About $£ 20000$ \\
\hline
\end{tabular}

^Using a standard endoscope to pull the sonde scope through the pylorus. laser or bipolar diathermy. In conclusion, push enteroscopy is a practical and valuable clinical service, which should probably become available on a subregional basis.

(Gut 1995; 37: 346-352)

Keywords: enteroscopy, small intestine, gastrointestinal bleeding, enteral nutrition

In life the small bowel is roughly three metres in length: at necropsy (and during enteroscopy) the organ may be stretched by 200-300\%. ${ }^{1}$ Mobile and tortuously folded in the peritoneal cavity, it is little surprise that endoscopy of this organ is technically challenging. Ten years after the first descriptions of non-operative enteroscopy ${ }^{2}$ it is perhaps surprising that so few centres in the United Kingdom are attempting the procedure. Many variations in technique and instrument design have been described ${ }^{3-5}$; however, at the current time, two types of endoscopes are available commercially for non-operative small bowel endoscopy. Push enteroscopy simply uses a long endoscope to permit passage beyond the second part of the duodenum by fairly standard endoscopic techniques. Purpose designed instruments have been developed (Olympus SIF-10/-10L), whose shaft length $(168 / 200 \mathrm{~cm}$ respectively) and characteristics of shaft rigidity, tip angulation, and optics are optimal for instrumentation of the small bowel. Although enteroscopy is possible using any long endoscope, adult colonoscopes are too cumbersome and rigid, and paediatric colonoscopes too flexible and usually too short (typically $135 \mathrm{~cm}$ ) to equal the performance of the purpose designed instruments. ${ }^{6}$ The alternative technique, sonde enteroscopy, uses an extremely flexible instrument the diameter of a bronchoscope $(5 \mathrm{~mm})$, and nearly three meters long (Olympus SSIF VII). ${ }^{7}$ The SSIF enteroscope is introduced through the nose, and usually pulled through the pylorus by means of a standard paediatric gastroscope passed orally. After passage through the pylorus, a distal balloon is inflated and the instrument propelled down the bowel by peristalsis. In contrast with push enteroscopy, both operator learning curve ${ }^{8}$ and procedure $^{9}$ are long, and heavy demands are made on staff and endoscopy unit time. Sonde enteroscopy is uncomfortable for the patient, ${ }^{9}$ and potentially dangerous, with a $3 \%$ perforation rate in one series. ${ }^{10}$ The instrument has no tip angulation controls or biopsy channel, and only an estimated $50-70 \%$ of the mucosa can be visualised during the instrument's 
withdrawal. ${ }^{9}$ Push and sonde enteroscopy are complementary, the first providing excellent views and therapeutic potential for a limited part of the small bowel and the second providing limited views and no therapeutic potential for most of the small bowel. Table I compares the features of push and sonde enteroscopy. We describe a three year experience of the purpose designed push type enteroscope (Olympus SIF-10) in routine clinical use, including instrumental techniques, and the wide range of both diagnostic and therapeutic purposes.

\section{Methods}

\section{Patients}

Sixty endoscopic procedures using the Olympus SIF-10 were undertaken specifically for small intestinal diagnostic or therapeutic purposes in 56 patients at the Royal London Hospital from July 1990 (date of instrument purchase) to March 1993. The instrument is also used for routine diagnostic gastroscopy when other instruments are unserviceable, for research purposes requiring small intestinal biopsies, and occasionally as a colonoscope in paediatric or tortuous colons: these examinations are not included in this report. The average procedure rate increased from 0.5 per month in 1990 to 4 per month in 1993. Patient demographics are described under appropriate results sections. Forty three studies were diagnostic (investigation of anaemia; abnormal barium studies suggesting upper small intestinal disorder; abdominal symptoms suggesting small intestinal mechanical lesion; abdominal symptoms suggesting malabsorption) and 17 were for attempted therapy, most commonly for difficult enteral feeding problems. The suitability of each patient for the investigation was determined in each case by a consultant gastroenterologist.

\section{Operator}

Procedures were performed by endoscopists experienced in standard upper and lower endoscopy (consultants $(n=4), 37$ procedures; senior registrars (3), nine procedures; registrars (2), 14 procedures).

\section{Standard push enteroscopy technique}

All procedures were well tolerated under light benzodiazepine sedation, with or without lignocaine throat spray, and most carried out on routine endoscopy lists, without the need for special equipment or extra staff. Intubation, and examination of oesophagus, stomach, and proximal duodenum proceeded as for routine oesphagogastroduodenoscopy (OGD). Further passage of the instrument proceeded by techniques mostly familiar to any experienced endoscopist. The usual limitation to progress was instrument looping in the stomach, preventing forward movement of the tip. In contrast with previous reports ${ }^{611}$ we did not find fluoroscopy mandatory, or that two operators were required. Fluoroscopy was used mainly to assist in safe placement of percutaneous endoscopic jejunostomy (PEJ) tubes, and when using an overtube (see later), but was of little help in routine enteroscopy. A number of techniques helped to assist progress. Pressure over the upper abdomen was useful in most cases. A duodenal length overtube was used in eight cases to attempt to limit instrument looping in the stomach. The technique is similar to that previously reported by Shimizu et al. ${ }^{6}$ The overtube consists of a stiff but flexible proximal shaft and a softer distal section with radio-opaque markers. Lubricating the inside of the overtube with silicone spray is useful. The overtube is preloaded onto the enteroscope. Once the enteroscope tip is in the distal duodenum, the shaft is pulled back straight and the overtube advanced so that the flexible portion lies within the duodenum. The technique is sometimes difficult and complications have been reported ${ }^{12}$ : it should probably only be attempted under fluoroscopic control. An internal channel stiffener designed for use with colonoscopes was useful in preventing instrument looping in three cases, and an Eder Puestow-type guidewire used to assist in navigation of a bend in one case. In contrast with the usual experience with colonoscopy, continued pressure that at first failed to cause tip progress would often produce movement after a brief wait, or in combination with abdominal palpation. Final depth of small bowel intubation was estimated by straightening the instrument to remove the gastric loop and subtracting $60 \mathrm{~cm}$ from the length inserted.

\section{Direct technique}

A standard 9F Freka-PEG system (Fresenius Ltd, Runcorn, UK) was used. A superficial loop of jejunum was identified by transillumination. High quality screening facilities and an experienced radiologist were considered essential to avoid inadvertent puncture of colon. Small amounts of soluble contrast media helped to confirm the anatomy, and hyoscine (Buscopan) was routinely used prior to jejunal loop puncture. After cannulation of the jejunal lumen, the procedure continued as for standard PEG placement by the 'pull' technique. ${ }^{13}$ Patients are reintubated to check the PEJ site. PEJs inserted for malignant disease remained in situ until death; PEJs used for temporary feeding problems were snared and removed using the enteroscope without complication.

The median (range) depth of small intestinal intubation was $45(15-90) \mathrm{cm}$. Procedure time was not formally recorded in most cases, however, the quickest diagnostic examination took about 10 minutes, and the longest (therapeutic) procedure about 45 minutes.

\section{Results}

\section{Anaemia}

Chronic iron deficiency anaemia was the commonest diagnostic indication, with 18 patients investigated. Of these, seven were taking non- 
TABLE II Details of patients undergoing enteroscopy for investigation of anaemia

\begin{tabular}{|c|c|c|c|c|c|c|}
\hline Case & $\begin{array}{l}\text { Agel } \\
\text { sex }\end{array}$ & $\begin{array}{l}\text { Indications and previous } \\
\text { GI investigations }\end{array}$ & $\begin{array}{l}\text { Insertion }(\mathrm{cm}) \\
\text { past pylorus }\end{array}$ & $\begin{array}{l}\text { Macroscopic } \\
\text { findings }\end{array}$ & $\begin{array}{l}\text { Histological } \\
\text { examinations }\end{array}$ & Final outcome \\
\hline 1 & $70 M$ & $\begin{array}{l}\text { Previous investigations normal } \\
\quad(\mathrm{OGD} \text {; Co; BaFT; BaE (x2)) }\end{array}$ & NR & $\begin{array}{l}\text { Jejunal angiodys- } \\
\text { plasia }\end{array}$ & - & $\begin{array}{l}\text { YAG laser treatments, reduced } \\
\text { transfusion requirement }\end{array}$ \\
\hline 2 & $70 \mathrm{~F}$ & $\begin{array}{l}\text { Previous investigations normal } \\
\text { (OGD; } \mathrm{Si} ; \mathrm{Co})\end{array}$ & 50 & Normal & - & Undiagnosed \\
\hline 3 & $47 \mathrm{M}$ & $\begin{array}{l}\text { Previous investigations normal } \\
\text { (OGD(3); Co(2); BaFT; } \\
\text { Mek; RBS) }\end{array}$ & 35 & Normal & - & $\begin{array}{l}\text { Laparotomy: metastatic } \\
\text { deposits in distal jejunum }\end{array}$ \\
\hline 4 & $50 \mathrm{~F}$ & $\begin{array}{l}\text { Previous investigations normal } \\
\text { (OGD; Co) }\end{array}$ & 70 & Normal & Normal & Undiagnosed \\
\hline 5 & $35 \mathrm{~F}$ & $\begin{array}{l}\text { Previous investigations normal } \\
\text { (OGD; Co) }\end{array}$ & NR & Normal & - & $\begin{array}{l}\text { Menorrhagia (previously } \\
\text { undisclosed) }\end{array}$ \\
\hline 6 & $76 \mathrm{M}$ & $\begin{array}{l}\text { Known antral angiodysplasia } \\
\text { remained anaemic despite } \\
\text { treatment }\end{array}$ & 70 & $\begin{array}{l}\text { Jejunal angiodys- } \\
\text { plasia }\end{array}$ & - & $\begin{array}{l}\text { YAG laser treatments reduced } \\
\text { transfusion requirement }\end{array}$ \\
\hline 7 & $79 \mathrm{M}$ & $\begin{array}{l}\text { OGD normal. Facial telangiec- } \\
\text { tasia suggested possibility of } \\
\text { SI angiodysplasia }\end{array}$ & 40 & Normal & - & Undiagnosed \\
\hline 8 & $40 \mathrm{~F}$ & $\begin{array}{l}\text { Recurrent haematemesis (OGD } \\
\times 3 \text { normal) }\end{array}$ & NR & $\begin{array}{l}\text { Friable raised } \\
\text { ulcer in D4 }\end{array}$ & Leiomyosarcoma & Surgical resection \\
\hline 9 & $75 \mathrm{M}$ & Haematemesis. (OGD normal) & 30 & Normal & - & Undiagnosed \\
\hline 10 & $37 \mathrm{M}$ & Haematemesis. (OGD normal) & 10 & Bleeding DU & - & Diathermy: bleeding stopped \\
\hline 11 & $46 \mathrm{~F}$ & $\begin{array}{l}\text { OGD: duodenum beyond reach } \\
\text { of endoscope appeared } \\
\text { irregular }\end{array}$ & 40 & $\begin{array}{l}\text { Raised ulcer with } \\
\text { black base }\end{array}$ & $\begin{array}{l}\text { Metastatic } \\
\text { melanoma }\end{array}$ & Surgical resection \\
\hline
\end{tabular}

$\mathrm{OGD}=$ oesophagogastroduodenoscopy $\mathrm{Co}=$ colonoscopy; $\mathrm{Si}=$ sigmoidoscopy; $\mathrm{BaE}=$ barium enema; $\mathrm{BaFT}=$ barium meal and follow through; Mek=Meckel's scan; RBS=labelled red cell scan; D3/D4=third/fourth part of duodenum; DU =duodenal ulcer; $\mathrm{NR}=$ data not recorded or not found

steroidal anti-inflammatory drugs (NSAIDs), and are considered separately later. The remaining 11 were not being treated with anticoagulants, aspirin or NSAIDs. Their average age (range) was 57 (33-79) years. Table II summarises the case details. Reasons for choosing enteroscopic examination were either: (a) source of gastrointestinal blood loss unclear despite multiple previous investigations, including at least one OGD and one colonoscopy (Table II, cases 1-5); or (b) limited number of normal previous investigations but a positive pointer towards a small intestinal source of bleeding (small intestinal angiodysplasias suspected on the basis of history or examination findings $(n=2)$; haematemesis $(n=3)$; distal duodenum distorted but beyond the reach of the standard gastroscope $(n=1)$ ) (Table II, cases 6 to 11). Including a previously missed (or perhaps newly developed) duodenal ulcer, a diagnosis was made as a result of enteroscopy in five of $11(45 \%)$ cases (Table II).

Seven patients (mean age (range) 58 (40-74) years) with inflammatory arthropathies, using NSAIDs on a regular basis, underwent enteroscopy for investigation of iron deficiency anaemia. All had previously been investigated by routine OGD: three patients remained anaemic three months after healing of gastric ulceration, two had only minor erosive lesions of stomach or duodenum, or both, and were thought likely to have other sites of blood loss to explain their anaemia, and two had a previously normal OGD. In view of the previously reported high incidence $(47 \%)$ of small intestinal ulceration in NSAID users ${ }^{14}$ enteroscopy was considered reasonable as an early investigation in these patients. The mean (range) instrument insertion past the pylorus was $67(50-90) \mathrm{cm}$. Minor abnormalities (a few mucosal red spots) were found in one patient, the remainder had normal macroscopic and microscopic upper small intestinal mucosa.

\section{Abnormal small bowel barium studies}

In nine patients the reason for enteroscopy was an abnormal small bowel barium examination. Table III summarises the results.

TABLE III Details of patients undergoing enteroscopy after abnormal barium follow through/small bowel enema findings

\begin{tabular}{|c|c|c|c|c|c|c|}
\hline \multicolumn{3}{|c|}{ Patient details } & \multicolumn{3}{|c|}{ Enteroscopic findings } & \multirow[b]{2}{*}{ Final outcome } \\
\hline & $\begin{array}{l}\text { Agel } \\
\text { sex }\end{array}$ & $\begin{array}{l}\text { Small bowel barium study } \\
\text { report/other patient details }\end{array}$ & $\begin{array}{l}\text { Insertion }(\mathrm{cm}) \\
\text { past pylorus }\end{array}$ & Macroscopic & Microscopic & \\
\hline 1 & $49 \mathrm{~F}$ & $\begin{array}{l}\text { Fixed deformity of distal duodenum with thickened } \\
\text { mucosal folds }\end{array}$ & 30 & Normal & Normal & $\begin{array}{l}\text { Barium study appearances } \\
\text { confirmed as artefact }\end{array}$ \\
\hline 2 & $33 \mathrm{M}$ & Abnormal mucosa in proximal jejunum & 40 & Normal & Normal & $\begin{array}{l}\text { Barium study appearances } \\
\text { confirmed as artefact }\end{array}$ \\
\hline 3 & $35 \mathrm{M}$ & $\begin{array}{l}\text { Abnormal mucosa in proximal jejunum - ?Crohn's } \\
\text { disease; slow transit patient with diabetes (NIDDM) }\end{array}$ & 45 & Normal & Normal & $\begin{array}{l}\text { Barium study appearances } \\
\text { confirmed as artefact }\end{array}$ \\
\hline 4 & $38 \mathrm{M}$ & $\begin{array}{l}\text { Distal small bowel mucosa thickened with shallow } \\
\text { ulcers; no stricturing: ?Crohn's }\end{array}$ & 50 & Duodenitis & $\begin{array}{l}\text { Non-specific duodenal and } \\
\text { jejunal inflammation }\end{array}$ & $\begin{array}{l}\text { Radiological diagnosis of } \\
\text { Crohn's disease }\end{array}$ \\
\hline 5 & $33 \mathrm{M}$ & $\begin{array}{l}\text { Thickened folds in upper small bowel/previous giardia } \\
\text { infection }\end{array}$ & 30 & $\begin{array}{l}\text { Thickened pale } \\
\text { mucosa }\end{array}$ & $\begin{array}{l}\text { Non-specific inflammation, } \\
\text { no giardia seen }\end{array}$ & Non-specific jejunitis \\
\hline 6 & $13 \mathrm{~F}$ & Small bowel mucosa coarse; non-specific appearances & NR & Normal & $\begin{array}{l}\text { Focal lymphoid aggregates } \\
\text { (normal for age); short villi }\end{array}$ & Lost to follow up \\
\hline 7 & $37 \mathrm{M}$ & Stricture in proximal jejunum & 35 (to lesion) & Stricture & $\begin{array}{l}\text { Acute inflammation with } \\
\text { granulomata }\end{array}$ & $\begin{array}{l}\text { Crohn's disease. Balloon } \\
\text { dilatation of stricture }\end{array}$ \\
\hline 8 & $58 \mathrm{M}$ & $\begin{array}{l}\text { Appearances suggest proximal small bowel } \\
\text { lymphoma/OGD normal }\end{array}$ & 30 & $\begin{array}{l}\text { Nodular, purple, } \\
\text { friable }\end{array}$ & Amyloid & Surgical resection \\
\hline 9 & $71 \mathrm{~F}$ & $\begin{array}{l}\text { Dilated upper jejunum proximal to stricture; } \\
\text { 'thumb-printed' antimesenteric border }\end{array}$ & 25 (to lesion) & $\begin{array}{l}\text { Ulcerated } \\
\text { stricture }\end{array}$ & Low grade lymphoma & $\begin{array}{l}\text { Symptomatic relief with } \\
\text { balloon dilatation } \star\end{array}$ \\
\hline
\end{tabular}

^TTS balloon-colonoscope length 'through the scope' balloon used through enteroscope biopsy channel. Abbreviations as in Table II. 
TABLE IV Details of patients undergoing enteroscopy for investigation of abdominal pain/vomiting

\begin{tabular}{|c|c|c|c|c|c|c|}
\hline \multicolumn{3}{|c|}{ Patient details } & \multicolumn{3}{|c|}{ Enteroscopic findings/diagnoses } & \multirow[b]{2}{*}{ Final outcome/diagnosis } \\
\hline & $\begin{array}{l}\text { Agel } \\
\text { sex }\end{array}$ & $\begin{array}{l}\text { History/previous } \\
\text { normal investigations }\end{array}$ & $\begin{array}{l}\text { Insertion }(\mathrm{cm}) \\
\text { past pylorus }\end{array}$ & Macroscopic & Microscopic & \\
\hline 1 & $41 \mathrm{~F}$ & $\begin{array}{l}\text { Recent subtotal gastrectomy for adenocarcinoma, } \\
\text { vomiting, ?obstruction }\end{array}$ & $\begin{array}{l}40 \text { (Past } \\
\text { anastomosis) }\end{array}$ & No obstruction & $\begin{array}{l}\text { Recurrence of } \\
\text { tumour }\end{array}$ & Chemotherapy \\
\hline 2 & $57 \mathrm{M}$ & Small bowel obstructive symptoms & 15 (to lesion) & Stricture - D3 & & Carcinoma of pancreas \\
\hline 3 & $66 \mathrm{~F}$ & $\begin{array}{l}\text { Small bowel obstructive symptoms, abdominal } \\
\text { mass/OGD }\end{array}$ & 80 & $\begin{array}{l}\text { DU, narrowed duodenum, } \\
\text { jejunal mucosa abnormal }\end{array}$ & Lymphoma & Resection \\
\hline 4 & $33 \mathrm{~F}$ & $\begin{array}{l}\text { Longstanding recurrent small bowel obstructive } \\
\text { symptoms }\end{array}$ & NR & Dilated lumen '?inflamed' & Normal & Pseudo-obstruction \\
\hline 5 & $57 \mathrm{M}$ & $\begin{array}{l}\text { Small bowel obstructive symptoms for } 6 \\
\text { months/OGD, BaFT, BaE, US, Lap }\end{array}$ & 70 & $\begin{array}{l}\text { Dilated lumen normal } \\
\text { mucosa }\end{array}$ & Normal & No diagnosis made \\
\hline 6 & $18 \mathrm{M}$ & $\begin{array}{l}\text { Abdominal pain, suspected functional symptoms but } \\
\text { loosing weight/OGD } \times 2 \text { ERCP, Co, Lap }\end{array}$ & NR & Normal & - & Functional symptoms \\
\hline 7 & $19 M$ & Chronic vomiting, thought to be functional/OGD & 80 & Normal & Normal & $\begin{array}{l}\text { Assisted in diagnosis of } \\
\text { functional cause }\end{array}$ \\
\hline
\end{tabular}

US=ultrasonography, Lap=laparoscopy or laparotomy. Other abbreviations as in Table II.

Enteroscopy provided clinically useful information in all cases. In cases 1-3 the region of abnormality suggested by the barium examination was reached, and was macroscopically and histologically normal, confirming the clinical impression that the barium appearances were artefactual. In cases 4-9 the barium study appearances were supported by abnormal small bowel macroscopic appearance or histological examination, or both. Histological examination of endoscopically obtained small intestinal biopsy specimens provided a definitive diagnosis in one third of examinations (cases 7-9, Table III).

\section{Abdominal symptoms}

Seven patients were studied because of abdominal pain or vomiting or both suggestive of small intestinal obstruction: in this group enteroscopy was performed either before small bowel radiology or after radiology was unhelpful. Table IV presents the details. Malignant lesions causing upper small intestinal obstruction were found in three patients (cases 1-3, Table IV), leading to appropriate treatment. Case 1 could have been diagnosed using a standard gastroscope, however, the enteroscope was used as the initial investigation because of the possibility of tumour recurrence involving jejunum beyond the reach of the standard gastroscope. In the remaining four patients a negative result assisted in the diagnosis of functional/motility disorders.

\section{Diarrhoea/suspected malabsorption}

Eight patients underwent enteroscopy because of diarrhoea undiagnosed by routine investigations, or where malabsorption was the suspected diagnosis. Table $\mathrm{V}$ shows the details. A tissue diagnosis was made as a result of enteroscopically obtained small intestinal biopsy specimens in two cases (Table V, cases 1 and 2), two patients had small intestinal lesions beyond the reach of the push enteroscope, eventually diagnosed radiologically; in two cases coeliac disease was excluded and in two the investigation was unhelpful. In case 2 the diagnosis could have been made on standard distal duodenal biopsies. Enteroscopy added further information, however, showing the distal extent of the ulceration and allowing more extensive biopsies of the ulcerated bowel.

\section{Therapeutic enteroscopy}

Fifteen patients, including one previously diagnosed by enteroscopy (Table III, case 9) underwent enteroscopy with the prior intention of therapeutic intervention, most commonly to establish an enteral feeding route where conventional techniques were inappropriate or had failed (10 cases). Six patients had potentially short term enteral feeding requirement. They were at high risk of aspiration with intragastric feeding, and were treated by jejunal feeding tubes placed over an $0.35 \mathrm{~mm}$ guidewire placed distal to the duodenojejunal flexure during enteroscopy (Table VI, cases 1-6). One patient, acutely unwell with chronic

TABLE V Details of patients undergoing enteroscopy for investigation of diarrhoea/malabsorption

\begin{tabular}{|c|c|c|c|c|c|c|}
\hline \multicolumn{3}{|c|}{ Patient details } & \multicolumn{3}{|c|}{ Enteroscopic findings/diagnoses } & \multirow[b]{2}{*}{ Final outcome } \\
\hline & $\begin{array}{l}\text { Agel } \\
\text { sex }\end{array}$ & History & $\begin{array}{l}\text { Insertion }(\mathrm{cm}) \\
\text { past pylorus }\end{array}$ & Macroscopic & Microscopic & \\
\hline 1 & $60 \mathrm{~F}$ & Diarrhoea, previous abdominal radiotherapy & 75 & Normal & $\begin{array}{r}\text { Radiation } \\
\text { jejunitis }\end{array}$ & Radiation jejunitis \\
\hline 2 & $69 \mathrm{~F}$ & Diarrhoea, anaemia & NR & $\begin{array}{l}\text { Severe distal duodenal/ } \\
\text { jejunal ulceration }\end{array}$ & $\begin{array}{l}\text { Coeliac } \\
\text { disease }\end{array}$ & $\begin{array}{l}\text { Responded to corticosteroids and } \\
\text { gluten free diet }\end{array}$ \\
\hline 3 & $68 \mathrm{M}$ & $\begin{array}{l}\text { Abdominal pain, diarrhoea, weight loss, anaemia. } \\
\text { Previous Kaposi's sarcoma of leg (HIV - ve) }\end{array}$ & 25 & Normal & $\begin{array}{l}\text { Jejunitis (non- } \\
\text { specific) }\end{array}$ & $\begin{array}{l}\text { Barium studies showed jejunocolonic } \\
\text { fistula, resection tissue histology - } \\
\text { Kaposi's sarcoma }\end{array}$ \\
\hline 4 & $23 \mathrm{M}$ & Malabsorption & 77 & Normal & Normal & $\begin{array}{l}\text { Radiology suggested distal small bowel } \\
\text { Crohn's disease }\end{array}$ \\
\hline $\begin{array}{l}5 \\
6 \\
7\end{array}$ & $\begin{array}{l}12 \mathrm{~F} \\
13 \mathrm{~F}\end{array}$ & $\begin{array}{l}\text { Strong family history of coeliac disease } \\
\text { Strong family history of coeliac disease }\end{array}$ & $\begin{array}{l}\text { NR } \\
\text { NR } \\
60\end{array}$ & Normal & $\begin{array}{l}\text { Normal } \\
\text { Normal }\end{array}$ & $\begin{array}{l}\text { Coeliac disease excluded } \\
\text { Coleiac disease excluded } \\
\text { Undiagnosed }\end{array}$ \\
\hline & & $\begin{array}{l}\text { Diarrhoea (known CLL and hypogammaglobuli- } \\
\text { naemia) }\end{array}$ & 60 & Normal & Normal & Undiagnosed \\
\hline 8 & $67 \mathrm{~F}$ & Diarrhoea & 25 & Normal & Normal & Undiagnosed \\
\hline
\end{tabular}

$\mathrm{CLL}=$ chronic lymphatic leukaemia. 
TABLE VI Therapeutic procedures attempted with the push enteroscope

\begin{tabular}{|c|c|c|c|}
\hline & Age/sex & History/therapeutic problem & Enteroscopic therapy \\
\hline 1 & $49 \mathrm{~F}$ & $\begin{array}{l}\text { Oesophageal reflux after Nissen fundoplication for scleroderma; nasogastric } \\
\text { feeding increased reflux }\end{array}$ & Nasojejunal feeding tube placed over guidewire \\
\hline 2 & $66 \mathrm{~F}$ & Recurrence of gastric carcinoma, gastric outlet obstruction & Nasojejunal feeding tube placed over guidewire \\
\hline 3 & $81 \mathrm{~F}$ & Duodenal stenosis secondary to pancreatic neoplasm & Nasojejunal feeding tube placed over guidewire \\
\hline 4 & $33 \mathrm{~F}$ & $\begin{array}{l}\text { Hereditary myopathy - acute exacerbation with myolysis, unable to feed - } \\
\text { high energy diet required for treatment }\end{array}$ & Nasojejunal feeding tube placed over guidewire \\
\hline 5 & $65 \mathrm{M}$ & $\begin{array}{l}\text { Metastatic carcinoma of bladder. Confusion, dehydration, and poor } \\
\text { nutrition. Repeatedly displaced nasogastric tubes }\end{array}$ & Nasojejunal feeding tube placed over guidewire tolerated \\
\hline 6 & $45 \mathrm{~F}$ & $\begin{array}{l}\text { Renal failure patient on CAPD, presenting with TB, SLE, and persistent } \\
\text { vomiting. Vomited up nasogastric and fine bore nasojejunal tubes }\end{array}$ & $\begin{array}{l}\text { Jejunal feeding tube placed over guidewire. Secured in distal } \\
\text { oesophagus by 'stitch' placed using an endoscopic sewing machine }\end{array}$ \\
\hline 7 & $68 \mathrm{M}$ & Gastric lymphoma and outlet obstruction. Vomiting and aspiration & $\begin{array}{l}\text { Insertion of double lumen feeding tube to enable jejunal feeding and } \\
\text { gastric aspiration immediately before surgery }\end{array}$ \\
\hline 8 & $60 \mathrm{~F}$ & Leaking anastomosis after laparoscopic oesophagectomy & PEJ (under general anaesthetic) \\
\hline 9 & $26 \mathrm{M}$ & Vomiting after gastric surgery (see text) & PEJ \\
\hline 10 & $55 \mathrm{M}$ & Carcinoma of oesophagus, intolerant of nasogastric feeding & PEJ \\
\hline 11 & $21 \mathrm{M}$ & Peutz-Jeghers syndrome, anaemia & Resection of $2.5 \mathrm{~cm}$ jejunal polyp \\
\hline 12 & $75 \mathrm{M}$ & Jaundice, attempt to find hepatojejunostomy & Technically unsuccessful \\
\hline 13 & $71 \mathrm{~F}$ & Friable stricture of jejunum diagnosed enteroscopically (Table II, case 9) & Balloon dilated on three separate occasions \\
\hline 14 & $75 \mathrm{~F}$ & Duodenal stricture secondary to carcinoma of pancreas & Balloon dilatation \\
\hline 15 & $76 \mathrm{~F}$ & $\begin{array}{l}\text { Known antral and duodenal angiodysplasia - for laser therapy of residual } \\
\text { small intestinal lesions }\end{array}$ & No further angiodysplastic lesions seen \\
\hline
\end{tabular}

$\mathrm{CAPD}=$ continuous ambulatory peritoneal dialysis; $\mathrm{TB}=$ tuberculosis; $\mathrm{SLE}=$ systemic lupus erythematosus.

renal failure, systemic lupus erythematosus and pulmonary tuberculosis, had been vomiting violently for several days, retching up nasogastric and nasojejunal tubes. The patient had a stitch placed in her distal oesophagus using an endoscopic sewing machine, to which an enteroscopically placed feeding tube was secured. This enabled nutritional intake throughout her illness, and the patient eventually recovered. Fine bore nasojejunal tube feeding was technically successful in all other cases, and continued until patients recovered sufficiently to tolerate normal feeding, or until surgery to relieve mechanical obstruction.

Three patients (Table VI, cases 8-10) had a PEJ inserted for treatment of longterm nutritional problems. In case 8 PEG was inappropriate because of a leaking gastrooesophageal junction following oesophagectomy: during PEJ feeding the anastomosis healed successfully without further surgery. Case 9 was a 26 year old man with frequent vomiting and failure to maintain weight after ulcer surgery. Multiple attempts at nasogastric feeding had failed, no tube staying in place for longer than 12 hours. A feeding tube was sewn into the stomach using the enteroscopic sewing machine, with the tip in the jejunum: although it was not displaced, feed was still vomited. A direct PEJ inserted using the push enteroscope was technically successful, and contrast studies showed free flow of contrast into jejunum and on to the colon. The patient, however, experienced retching and 'unbearable' pain at the site of the jejunostomy whenever PEJ feeding was attempted. The patient was therefore given intravenous nutrition. In case 10 the patient could not tolerate nasogastric or PEG feeding: PEJ permitted adequate nutritional input without vomiting.

Other successful therapeutic procedures (Table VI) were a polypectomy in a patient with Peutz-Jeghers syndrome (using standard monopolar diathermy equipment) and balloon dilatation of strictures. Additionally, bleeding lesions detected in the obscure anaemia diagnostic group (Table II) were successfully treated using bipolar diathermy and YAG laser techniques.

\section{Discussion}

We found push enteroscopy an easy and useful technique for the investigation of small intestinal disease, and of particular value for therapeutic procedures where upper small intestinal access was required. Of 43 patients undergoing diagnostic enteroscopy, a positive diagnosis was made in $12(29 \%)$ cases, although in two of these the diagnosis could have been made by routine OGD. The highest positive diagnostic rates were in patients with unexplained anaemia (excluding NSAID users) $(45 \%)$, or with abnormal small bowel barium studies $(33 \%)$. Additionally, we successfully treated all 10 patients referred with difficult feeding problems by enteroscopic placement of jejunal feeding devices, and were able to carry out stricture dilatations, laser treatment of angiodysplasias and polypectomies in the upper small bowel.

Most previous studies in published reports concern the use of enteroscopy for the investigation of obscure gastrointestinal bleeding. ${ }^{3-5810111415-18}$ This is an important clinical problem: of all acute gastrointestinal bleeds $10-20 \%$ cases remain undiagnosed at time of initial bleeding and $1-6 \%$ become recurrent but remain undiagnosed despite multiple investigations of the upper and lower gastrointestinal tract. ${ }^{16}$ Although intraoperative enteroscopy is considered the technical gold standard for small bowel examination, it is difficult to compare results with non-operative techniques because of different selection criteria. Few surgeons would consider laparoscopy and intraoperative enteroscopy until the case for a small intestinal site of disorder is confirmed ${ }^{16}{ }^{19}$ : not surprisingly, small intestinal lesions are detected in most cases in such series. ${ }^{19} 20$ The lower success rates of the less invasive push and sonde techniques is in part due to their earlier use in the investigation of obscure gastrointestinal bleeding cases. Using push enteroscopy with adult colonoscopes, the source of obscure gastrointestinal blood loss has been detected in $17-46 \%$ cases. ${ }^{311}$ Using sonde enteroscopy in similar cases, success rates have been comparable, ranging from $26-38 \% .^{81015}$ Reports of sonde enteroscopy in subgroups who eventually proceeded to 
operative enteroscopy not surprisingly show higher success rates. ${ }^{18}$ The similar detection rates of push and sonde techniques presumably reflects the diffuse nature of angiodysplastic lesions, the commonest overall cause of obscure gastrointestinal bleeding. ${ }^{21}$

In seven anaemic patients taking NSAID drugs we found only one upper small intestinal lesion, of dubious significance: this contrasts with the $47 \%$ incidence of small intestinal ulceration previously reported. ${ }^{14}$ This discrepancy may result from less rigorous selection of our patients (not all had normal upper endoscopy, and only two had had colonoscopy), or perhaps as such lesions seem to be mostly common in the distal small bowel (K J F MacKenzie, personal communication), thus beyond the reach of the push instrument.

In our diagnostic group as a whole, four (10\% cases) patients had malignancies (three primary, one secondary) involving the upper small intestine detected by push enteroscopy. In a study of 258 patients with obscure gastrointestinal bleeding investigated by sonde enteroscopy, small bowel tumours were the single most common cause in patients under 50 years. $^{22}$ The diagram (but not the text) in this report suggests that over half of the 13 tumours detected would be detected by push enteroscopy (but not routine gastroscopy). Interestingly, four of these tumours were missed by small bowel radiology. ${ }^{22}$ Thus there will be a small percentage of patients presenting with obscure gastrointestinal bleeding who have normal barium studies and push enteroscopy, but who have a small intestinal tumour. Sonde enteroscopy would seem the least invasive and most sensitive next investigation in such patients.

The investigation of patients with abnormal small bowel barium studies was an excellent indication for push enteroscopy, particularly for the tissue diagnosis of suspected malignant lesions. Barium studies are known to have a significant false negative rate for small intestinal malignancies. ${ }^{23}$ Our series also shows the problem of false positive reports, with three examples. Push enteroscopy and small bowel enema may be usefully combined at one sitting. ${ }^{24}$ This technique has the advantages that the initial enteroscopy views the small bowel proximal to the duodenojejunal flexure, which may be inadequately shown by enterocyclis, and that passage of the enema tube past the duodenojejunal flexure (which improves the quality of the examination) is made easier for radiologist and patient by a guidewire positioned enteroscopically under sedation.

Enteroscopy seemed less useful for investigating patients with abdominal pain or symptoms of upper small bowel obstruction, or both. Out of seven cases the only definitive result was the early tissue diagnosis of jejunal lymphoma. Small bowel enema has a $100 \%$ positive predictive value for small intestinal obstruction $^{25}$ and should probably precede enteroscopy in cases of suspected obstruction.

In a previous study ${ }^{26}$ jejunal biopsy specimens taken with a hydraulic capsule device were not diagnostically superior to duodenal 'pinch' biopsy specimens using a standard gastroscope. In theory, however, the interpretation of proximal duodenal biopsy specimens in cases of small intestinal disease may be hampered by the normal occurrence of flattened villi and Brunner's glands, and by duodenitis, often present but usually irrelevant to any malabsorption process. Coeliac disease is occasionally more prominent in the jejunum than the duodenum, and several small intestinal diseases have a patchy involvement (lymphoma, Crohn's disease, eosinophilic enteritis). Given these theoretical drawbacks of duodenal biopsy specimens, and from the experience in this and previous ${ }^{27}$ studies, it seems reasonable that enteroscopically targeted jejunal biopsy specimens should be the investigation of choice in suspected intestinal disease causing diarrhoea/malabsorption.

The treatment of difficult feeding problems was the most frequent therapeutic intervention with the enteroscope. Jejunal feeding is probably preferable to gastric as it reduces the risk of aspiration, an important problem with PEG. ${ }^{28-30}$ In this study temporary, fine bore feeding tubes, placed through the nose, were far more secure if positioned distal to the duodenojejunal flexure over an enteroscopically placed guidewire. In patients with recent oesophageal surgery, anastomotic leaks in the upper gut, severe reflux related problems, gastric dysmotility or gastric/duodenal outlet obstruction, jejunal feeding becomes the only safe enteral route. For longterm feeding directly into the jejunum, the technique of passing a feeding tube through a PEG, pulling it into the upper small bowel with an endoscope (JETPEG), is probably less satisfactory than the direct PEJ method we used. ${ }^{31}$ Tubes placed by the JETPEG method usually still lie proximal to the duodenal-jejunal flexure, and reflux will not be effectively prevented. JETPEG tubes are narrower than direct PEJ tubes, thus more prone to kinking and blockage. ${ }^{31}$

As far as we are aware, our series contains the first case of non-surgical polypectomy for control of small intestinal bleeding in PeutzJeghers syndrome (although the use of intraoperative polypectomy has been described). ${ }^{32}$ Dilatation of small intestinal strictures and laser treatment of angiodysplasias were completed successfully in this series. Push enteroscopy may also be useful for exploration of jejunal limbs loops after gastric surgery or hepatojejunostomy, and for ERCP with Roux-en-Y limbs. ${ }^{33}$

In conclusion, our study suggests that push enteroscopy is quick to do, not particularly difficult to learn, and practical for use in endoscopy units that currently offer a diagnostic and therapeutic service. The procedure has an acceptably wide range of uses and acceptably high diagnostic/therapeutic success rate to justify development of the service, probably on a subregional basis. Sonde or intraoperative enteroscopy, or both, should perhaps be reserved for selected cases not diagnosed by push enteroscopy and radiological imaging, and available on a regional basis. 
The enteroscope was taken to Oldchurch Hospital, Romford, Essex for investigation of patient ' 8 ' in Table II: we thank Dr R Burnham for permission to use data from this patient.

These data have been published as an abstract in Gut 1993;

34 (suppl 1): S30.

1 Morson BC, Dawson IMP. Gastro-intestinal pathology. 1st ed. Oxford, Blackwell Scientific, 1972: 183-205.

2 Parker HW, Agayoff JD. Enteroscopy and small bowel biopsy using a peroral colonoscope. Gastrointest Endosc 1983; 29: 139-40.

3 Lewis BS, Waye JD. Small bowel enteroscopy in 1988: pros and cons. Am $\mathcal{F}$ Gastroenterol 1988; 83: 799-802.

4 Bowden TA. Endoscopy of the small intestine. Surg Clin $N$ Am 1989; 69: 1237-47.

5 Gilbert DA, Buelow RG, Chung RSK, Cunningham JT, Foutch PG, Laine LA, et al. Status evaluation: enteroscopy. Gastrointest Endosc 1991; 37: 673-7.

6 Shimizu S, Tada M, Kawai K. Development of a new insertion technique in push-type enteroscopy. $A m \mathcal{J}$ tion technique in push-type

7 Tada M, Shimizu S, Kawai K. A new transnasal sonde type fiberscope (SSIF type VII) as a pan-enteroscope. Endoscopy 1986; 18: 121-4.

8 Gostout CJ, Schroeder KW, Burton DD. Small bowel enteroscopy: an early experience in GI bleeding of unknown origin. Gastrointest Endosc 1991; 37: 5-8.

9 Lewis BS, Waye JD. Total small bowel enteroscopy. Gastrointest Endosc 1987; 33: 435-7.

10 Morris AJ, Wasson LA, MacKenzie JF. Small bowel enteroscopy in undiagnosed gastrointestinal blood loss. Gut 1992; 33: 887-9.

11 Foutch PG, Sawyer R, Sanowski RA. Push-enteroscopy for diagnosis of patients with gastrointestinal bleeding of diagnosis of patients with gastrointestinal bleeding

12 Barkin JS, Reiner DK, Lewis BS, Waye JD, Goldberg RI, Phillips R. Diagnostic and therapeutic jejunoscopy with the SIF-10L: longer is really better. [Abstract]. Gastrointest Endosc 1990; 36: 214

13 Shike M, Wallach C, Likier H. Direct percutaneous endoscopic jejunostomies. Gastrointest Endosc 1991; 37: 62-5.

14 Morris AJ, Madhok R, Sturock RD, Capell HA, MacKenzie JF. Enteroscopic diagnosis of small bowel ulceration in patients receiving non-steroidal anti-inflammatory drugs. Lancet 1991; 337: 520.

15 Lewis B, Wayne J. Gastrointestinal bleeding of obscure origin: the role of small bowel enteroscopy. origin: the role of small

$16 \mathrm{Lau}$ WY, Yuen WK, Chu KW, Poon GP, Li AKC. Obscure bleeding in the gastrointestinal tract originating in the small intestine. Surg Gynecol Obstet 1992; 174: $119-24$

17 Lewis BS, Wegner JS, Waye JD. Small bowel enteroscopy and intraoperative enteroscopy for obscure gastrointestinal bleeding. Am f Gastroenterol 1991; 86: 171-4.

18 Szold A, Katz LB, Lewis BS. Surgical approach to occult gastrointestinal bleeding Am $\mathcal{f}$ Surg 1992; 163: 90-3

19 Desa LA, Ohri SK, Hutton KAR, Lee H, Spencer J. Role of interoperative enteroscopy in obscure gastrointestinal interoperative enteroscopy in obscure gastrointestinal

20 Ress AM, Benacci JC, Sarr MG. Efficacy of intraoperative enteroscopy in diagnosis and prevention of recurrent gastrointestinal bleeding. Am f Surg 1992; 163: 94-9.

21 Gostout CJ. Improving the withdrawal phase of Sonde enteroscopy with the 'push away' method. Gastrointest Endosc 1993; 39: 69-72.

22 Lewis BS, Kornbluth A, Waye JD. Small bowel tumours: yield of enteroscopy. Gut 1991; 32: 763-5.

23 Martin LF, Max MH, Richardson JD, Peterson GH Small bowel tumours: a continuing challenge. South Med $\mathcal{f}$ 1980; 73: 981-5.

24 Cohen ME, Barkin JS. Enteroscopy and enteroclysis: the combined procedure. Am $\mathcal{f}$ Gastroenterol 1989; 84: $1413-5$

25 Sharke PD, Rex DK, Lappas JC, Maglinte DDT. Radiographic evaluation of suspected small bowel obstruction. Am f Gastroenterol 1991; 86: 175-8.

26 Scott BB, Jenkins D. Endoscopic small intestinal biopsy. Gastrointest Endosc 1981; 27: 162-7.

27 Barkin JS, Schonfield W, Thomsen S, Manten HD, Rogers AI. Enteroscopy and small bowel biopsy - an improved technique for the diagnosis of small bowel disease. Gastrointest Endosc 1985; 31: 215-7.

28 Burtch G, Shatney C. Feeding gastrostomy. Assistant or assassin. Am Surg 1985; 51: 204-7.

29 Cole M, Smith J, Molnarc, Shaffer E. Aspiration after percutaneous gastrostomy. Assessment by Tc-99m labeling cutaneous gastrostomy. Assessment by Tc-99m label

30 Hassett J, Sunby C, Flint L. No elimination of aspiration pneumonia in neurologically disabled patients with feeding gastrostomy. Surg Gynecol Obstet 1988; 167: 383-8.

1 Lewis BS. Perform PEJ, not PED. Gastrointest Endosc 1990; 36: 311-3.

32 Spigelman AD, Thompson JPS, Phillips RKS. Towards decreasing the relaparotomy rate in Peutz-Jeghers syndrome: the role of peroperative small bowel endoscopy $B r$ f Surg 1990; 77: 301-2.

33 Gostout CJ, Bender CE. Cholangiopancreatography, sphincterotomy, and common duct stone removal via sphincterotomy, and common duct stone removal via 156-63. 